\title{
Steroid-Transforming Microorganisms: Beneficial Microbes for Biotechnology and Medicine
}

\section{Marina Donova*}

Institute of Biochemistry and Physiology of Microorganisms, Russian Academy of Sciences, Russian Federation, Russia

*Corresponding Author: Marina Donova, Institute of Biochemistry and Physiology of Microorganisms, Russian Academy of Sciences, Russian Federation, Russia.

Received: August 27, 2018; Published: November 01, 2018

DOI: 10.31080/ASMI.2018.01.0127

Steroids are the terpenoid lipids that contain gonane core of the four fused cycloalkane rings (A-D). The superfamily of steroids includes sterols (such as cholesterol, sitosterol, ergosterol, campesterol, etc.), bile acids (e.g. cholic, ursodeoxycholic, ursocholic, deoxycholic, lithocholic acids, etc.), corticoids (cortisol), mineralocorticoids (aldosterone), cardiac aglycones, vitamin D, and others. Steroids perform essential vital functions in all living organisms of the animal and plant kingdom, being signal molecules as sex hormones in the reproduction of vertebrates (androgens, estrogens, progestagens), adrenal hormones (cortisol), provide electrolyte and glucose homeostasis in higher organisms, regulate signal transduction pathways by the binding to the respective intracellular receptors, function as allosteric modulators of neurotransmitter receptors. Bile acids play key role in the digestion and re-sorption of fats, fatty acids and lipid-soluble vitamins in the digestive tracts of vertebrates, participate in cholesterol balancing, apoptosis, colonic salvage, and serve other important physiological functions. In vertebrates, the hormones, bile acids and other essential steroids are synthesized from cholesterol.

Important biological roles of cholesterol and other sterols include also cell membrane stabilization and providing of membrane fluidity. Cholesterol is known as "animal” sterol. Its structural analogs are phytosterols, - a mixture of plant sterols (sitosterol, stigmasterol, campesterol, brassicasterol, etc.). Ergosterol is an essential component of low eukaryote cell wall and serves as chemical marker of the fungal contamination.

Steroids were reputed to have originated hundreds of millions of years ago. They are regarded as chemical fossils and often used as geochemical markers in the sediment maturity estimation in petroleum geochemistry. Throughout evolution microorganisms have been exposed to variety steroid substrates resulting in numerous metabolites and enzymatic activities.
Bacteria from different habitats (soil, aquatic environments, eukaryote hosts etc.) were evolved to utilize steroids as ubiquitous growth substrates. Recent metagenome studies confirmed that steroid-degrading bacteria are globally distributed and prevalent in wastewater treatment plants, soil, plant rhizospheres, and the marine environment [1]. 265 putative steroid degraders have been identified within only Actinobacteria and Proteobacteria whose genomes are available in NCBI (NCBI Rf_Seq) [2].

During the last two decades, a new concept was emerged in biomedical sciences considering the microbiome of vertebrates as a virtual 'organ' with endocrine function, e.g. [3]. The interaction between the mammalian microbiome and the hormonal milieu influences age and sex differences, immune function and the pathogenesis of diseases. There is growing body of research evidencing a role of microbiota in the microbial metabolism of endogenous adrenocorticosteroids and blood pressure regulation as a contributing factor in the etiology of essential hypertension. Multiple mechanisms for regulation of host hormone production and receptor signaling were evolved by commensal and pathogenic microbes to promote their growth, survival, and transmission. The data were published to illustrate that gut microbiota relates to obesity, diabetes and the metabolic syndrome [4]. Microbiome-steroid interactions studies are of great importance for the medicine and veterinary: unlike the host genome, the microbiome "second genome" can be altered by diet or microbiota transplantation to the host thus providing new prospects for biomedicine. New revolutionary findings in the field are expected in the near future.

In addition to effects on host immune responses during infection, steroids can directly affect and even be utilized by microbes [4]. For example, the known intracellular pathogens such as $M y$ cobacterium tuberculosis and Rhodococcus equi assimilate cholesterol directly from the host organism and the enzymes involved 
in cholesterol degradation pathway are considered as drug targets against the heavy infections caused by the pathogens [5].

Unlike bacteria, fungi which are the most diverse kingdom of eukaryotes are generally incapable of full steroid degradation but detoxify steroids as fungitoxic compounds. Key features of filamentous fungi are their capability to steroid oxyfunctionalization such as hydroxylation, or lactonization. As compared to mammalian ones, fungal hydroxylase systems often exhibit higher catalytic rates, and some of the cost effective and reliable hydroxylation bioprocesses catalyzed by fungal strains have been implemented at the industrial level (e.g. the adrenocortical hormones production). Knowledge of the peculiarities of bioconversion of natural and synthetic steroids by fungi is also important for preclinical studies (the direction is known as "microbial models of mammalian metabolism").

The capability of microorganisms to modify steroids is widely used in biotechnology mainly for production of pharmaceutical steroids, or key precursors for their synthesis, Steroid compounds are now ranked among the most marketed products of pharmaceutical industry with annual global market exceeding 10 billion US dollars, and microbially derived steroids represent considerable part of it. For example, aerobic degradation of phytosterol side chain with actinobacteria (mainly, selected mutant or recombinant strains of non-pathogenic mycobacteria such as Mycobacterium neoaurum is a base for industrial production of androstane steroids which are the starting molecules for various synthetic routes of steroid hormones. The selected strains of actinobacteria such as Arthrobacter simplex (Pimelobacter simplex, Nocardioides simplex) are used for production of 1-dehydrosteroids (prednisolone, methylprednisolone, exemestane). Effective production schemes for ursodeoxycholic acid include microbial and enzymatic stages. There is increasing evidence of growing biotechnology applications of steroid-transforming microorganisms in pharmaceutical industry and related fields (environmental protection from endocrine disrupting compounds, veterinary, agrochemistry).

Microbial diversity is huge, and the metabolic capabilities of microorganisms can be more enhanced by genetic engineering and recombinant techniques to provide an inexhaustible source of bioactive molecules. The progress in genetic and metabolic engineering opened a new era in steroid biotechnology. New genetically modified microorganisms are being created capable of selective performing various reactions of steroid transformation [6]. However, low productivity of strains, their insufficient stability and some problems in the bioprocesses scale-up often restrict their commercial application and many brilliant developments still do not overcome the laboratory level. Solving these and many other problems requires a fundamental knowledge, a deep scientific basis to create a new generation of biocatalysts and innovative bioprocesses for the pharmaceutical industry of steroids.

Therefore, research on microbial steroid transformation is important on many reasons: we have to more clearly understand a role of the microbiome in steroid functioning and circulation in human body to treat infections, hypertension, obesity, metabolic disorders, cancer, and other diseases and predict microbial response to steroids exposures. On the other hand, study of microbial diversity and metabolic capabilities of microorganisms, discovery and creation of novel industrial biocatalysts for effective production of value-added steroids is required for ecofriendly and cost-effective biotechnologies development.

The novel findings in the field will contribute to the solving of major challenges of the contemporary society such as life quality, healthy development and ageing.

\section{Acknowledgements}

Russian Science Foundation is greatly acknowledged for the support (Grant No. 18-14-00361).

\section{Bibliography}

1. Holert J., et al. "Metagenomes reveal global distribution of bacterial steroid catabolism in natural, engineered, and host environments". MBio 9.1 (2018): e0234-e0217.

2. Bergstrand LH., et al. "Delineation of steroid degrading microorganisms through comparative genomic analysis". MBio 7 (2016): e00166.

3. Morris DJ and Ridlon JM. "Glucocorticoids and gut bacteria: The GALF Hypothesis" in the metagenomic era". Steroids 125 (2017): 1-13.

4. Steeg LG and Klein SL. "Sex steroids mediate bidirectional interactions between hosts and microbes". Hormones and Behavior 88 (2017): 45-51.

5. Pandey AK and Sassetti CM. "Mycobacterial persistence requires the utilization of host cholesterol". Proceedings of the National Academy of Sciences of the United States of America 105 (2008): 4376-4380.

6. Fernandez-Cabezon L., et al. "New insights on steroid biotechnology”. Frontiers in Microbiology 9 (2018): 958.

\section{Volume 1 Issue 12 December 2018 (C) All rights are reserved by Marina Donova.}

